Bio-grafía: Escritos sobre la Biologia y su Enseñanza Vol 4 No6 ISSN 2027-1034. Primer semestre de 2011, Bogotá, Colombia, pp 191-204

\title{
OBSERVANDO NUESTRO ENTORNO LOCAL: EXPLORACIONES ESCOLARES EN LA ZONA CENTRO NORTE DE CHILE.
}

\section{WATCHING OUR LOCAL ENVIRONMENT: SCHOOL EXPLORATIONS IN THE ZONE CENTER NORTH OF CHILE.}

Por: César Piñones Cañete ${ }^{1}$

Recibido: 15 -03- 2011

Aceptado: 03-06-2011

\section{Presentación de la Experiencia:}

El presente trabajo es una síntesis de dos procesos de indagación naturalista, desarrollados con jóvenes de enseñanza secundaria, en el marco del currículum de estudio de la asignatura de biología definidos para Chile. Se diseñó e implementó un conjunto de actividades que propiciaron la participación activa de los estudiantes, siendo ellos protagonistas de sus propios aprendizajes, por medio de la exploración de los espacios naturales cercanos a sus colegios.

Se busca con este artículo: a) describir las características del programa científico escolar ejecutado, b) documentar las acciones de indagación desarrolladas por los estudiantes y c) sintetizar los principales resultados pedagógicos de las experiencias.

\section{Primeras Consideraciones.}

La formación en la asignatura de Biología dentro del marco curricular chileno, establece para el tercer y cuarto año de enseñanza media (cursados por jóvenes con edades entre los 16 a 17 y 18 a 19 años respectivamente), planes de especialización de libre elección por parte de los estudiantes, en donde se profundizan temáticas de la disciplina desde el origen y evolución de la vida, hasta la relación del hombre y su entorno. En este contexto, en un intervalo de dos años, se eligió una de las unidades de estos planes de estudio, abordando el tratamiento de sus contenidos bajo la propuesta metodológica Enseñanza de la Ecología en el Patio de la Escuela, EEPE (Arango, et al. 2002).

Se pretendió con esto incorporar de forma central el método indagatorio en el desarrollo de las distintas categorías que componen las unidades, proponiendo como principal estrategia el trabajo de campo. Es así, como se guio a dos grupos distintos de alumnos y alumnas de la Región de Coquimbo, Chile, a través de actividades progresivas de estudio de la biodiversidad, desde el propio patio de

\footnotetext{
${ }^{1}$ Licenciado en Educación. Profesor de Biología y Ciencias Naturales. Asesor Red de Apoyo a la Conservación del Chinchilla, RACCH. autopoiesis.uls@gmail.com / www.redchinchilla.org. Fotografías por el autor.
} 
sus escuelas, hasta llegar a explorar e investigar algunas de las características ecológicas de un parque urbano y humedales de ambientes semiáridos de la zona centro norte de Chile (ver fotografías 1 y 2).

Ambas etapas de este proyecto, fueron socializadas en distintas jornadas entre grupos de pares docentes, tales como el Primer Encuentro Nacional de Divulgadores Científicos 2010 (Piñones, 2010) y el Segundo Encuentro Nacional de Educación Ambiental Habla Educad@r durante el mes de enero de 2011.
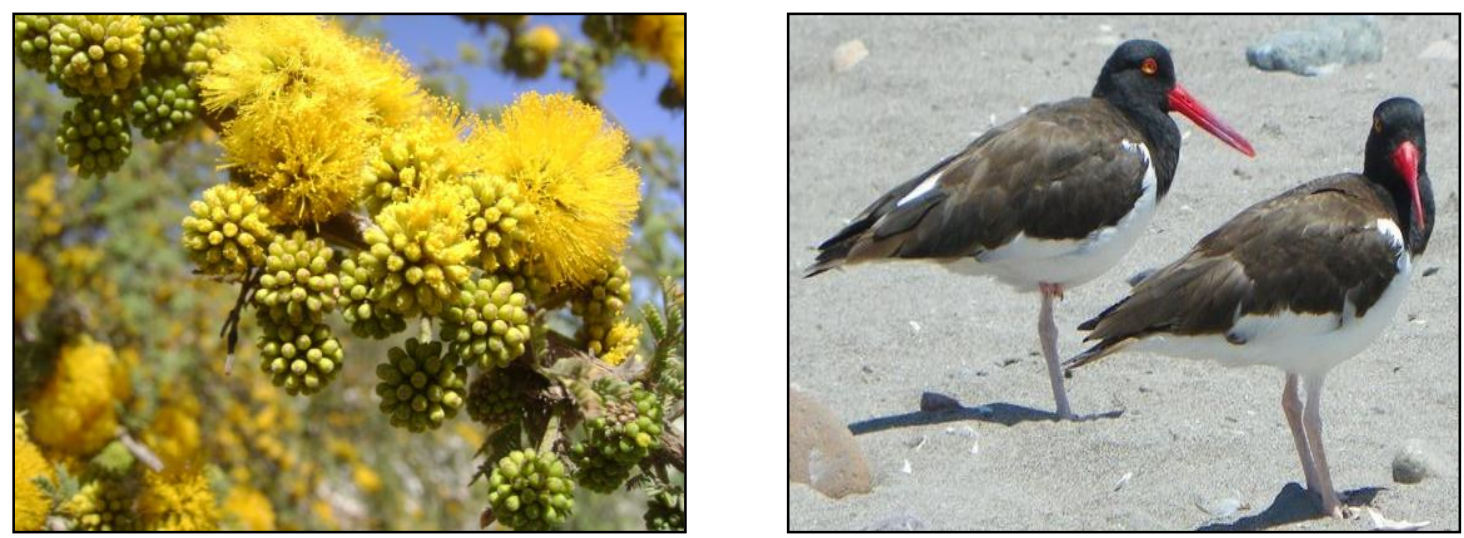

\section{Fotos 1 y 2: Biodiversidad de la Región de Coquimbo. Acacia caven, Espino, especie de valles y serranías \& Haematopus palliatus, Pilpilén común, ave costera.}

\section{Grupos de Trabajo.}

El primer equipo de estudiantes, estuvo conformado por 12 jóvenes que para el 2009, cursaban su última etapa de escolarización, correspondiente al cuarto año de enseñanza secundaria en el Colegio San Nicolás de la ciudad de La Serena, Provincia del Elquí. El plan de estudio de este nivel contempla tres unidades, de las cuales la denominada "Investigación Científica en Biología", fue abordada considerando el modelo EEPE.
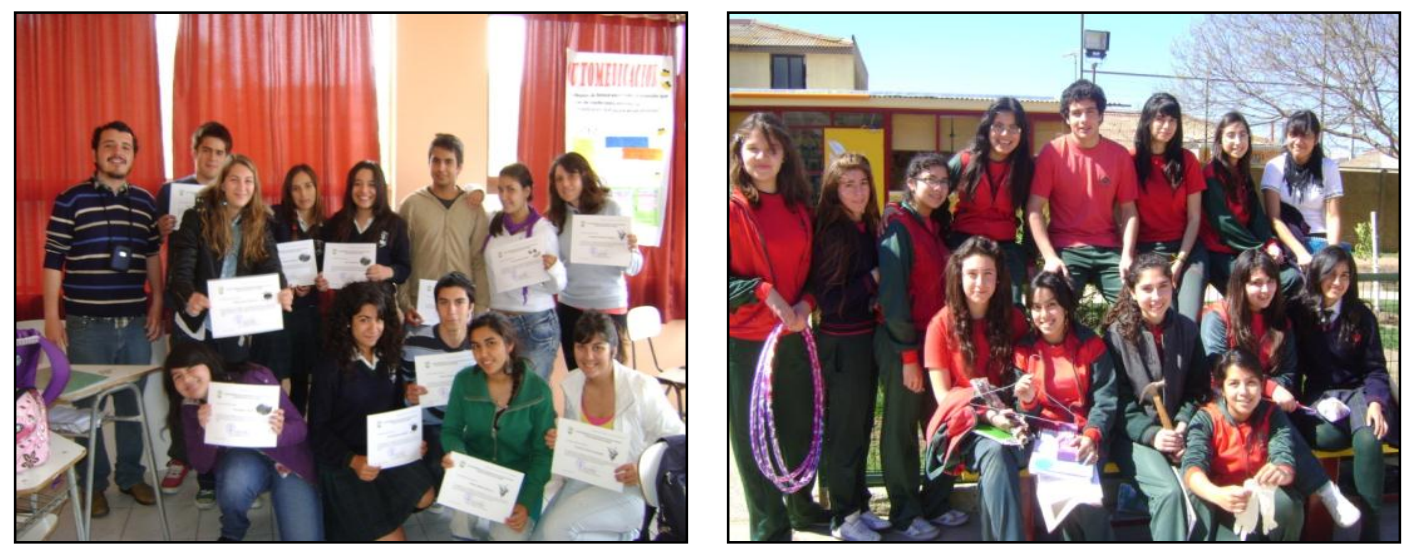

Fotos 3 y 4: Estudiantes Colegio San Nicolás, La Serena (izquierda) \& Estudiantes Colegio Pucará, Ovalle (derecha). 
Por su parte, durante el 2010 se trabajó con un grupo de 15 estudiantes, los cuales cursaban su tercer nivel de enseñanza media en el Colegio Pucará de la ciudad de Ovalle, Provincia del Limarí. De igual forma, de entre las tres unidades del plan de estudio, se les propuso abordar bajo los principios de la EEPE el tema "Flujo y procesamiento de energía y materia en los sistemas biológicos" (ver fotografías 3 y 4).

\section{Fundamentos del Proyecto.}

En el plano de la educación formal, tanto en la región de Coquimbo, como en otras regiones de Chile, se desarrollan actualmente variados programas escolares científicosambientales, muchos de los cuales tienen como común denominador el enfoque indagatorio. La gran mayoría de estas iniciativas se centran en grupos de estudiantes de los primeros niveles de enseñanza primaria. Por lo demás, en el último tiempo la educación parvularia o inicial se ha integrado a estos proyectos, desarrollándose experiencias interesantes referidas al estudio de la biodiversidad y otros aspectos del entorno natural y social, proyectos muchos de ellos financiados por fondos que entrega el estado a través de distintos programas de valoración y divulgación de la ciencia y educación ambiental.

Las experiencias ejecutadas en enseñanza secundaria bajo este enfoque metodológico con adolescentes son más bien escasas, lo cual no hace más que plantearnos un desafío, si pensamos en la necesaria continuidad y proyección de las ideas y principios que fundamentan estos esfuerzos de reformular las tradicionales pautas propedéuticas, con las que muchas veces se enseña las ciencias naturales.

Por lo demás, el marco curricular actual chileno pretende dar énfasis a las habilidades de investigación y el desarrollo de formas de observación, razonamiento y de proceder, características de una actitud científica, así como las de exposición, comunicación, discusión, debate y argumentación de resultados de actividades experimentales o de indagación (Ministerio de Educación, 2002).

No quedan fuera consideraciones en el plano de la educación ambiental, área de desarrollo transversal en el currículum, donde se plantea, por ejemplo, que la práctica educativa debe afianzar la protección del entorno natural y sus recursos como contexto de desarrollo humano (Ministerio de Educación, 2005)

Así el modelo indagatorio, en base a la metodología EEPE, se transforma en una interesante bisagra para articular dos importantes dimensiones educativas dentro del plano formal: la ambiental y científica. Esta propuesta ha sido desarrollada a partir de 1987 por científicos, profesores, estudiantes y educadores ambientales, tanto en Norteamérica como en Latinoamérica. La EEPE es aprender haciendo y reflexionando, mediante un proceso que vincula de forma inseparable la acción y la reflexión (Arango et al. 2009). 
Los programas pedagógicos desarrollados bajo este modelo, siguen un ciclo de indagación de primera mano por medio de la estrategia cíclica pregunta-acción-reflexión y surge como respuesta al complejo y muchas veces árido tratamiento de las ciencias a nivel escolar, en donde se muestra la actividad científica a través de un método (aparentemente único) lleno de aristas y sólo posible de ser comprendido por los científicos y científicas. La esencia del método científico en cualquiera de sus versiones involucra un proceso natural y de sentido común que mucha gente usa para darse cuenta de su vida cotidiana. Por lo tanto, no tiene que haber nada oculto y extraño sobre los científicos y sus métodos (Arango, et al. 2009).

Es a través de la apropiación de una secuencia sencilla pero fundamental de pasos, como se adquieren destrezas y actitudes propias de las indagaciones científicas, tales como la curiosidad, perseverancia y rigurosidad en la toma de datos, etc.

¿Qué opinión tienen los estudiantes frente a la enseñanza de la biología?

Previo al trabajo indagatorio, se aplicó un breve cuestionario, con miras a conocer opiniones y expectativas frente al proyecto propuesto y así incorporarlos en la evaluación de la iniciativa. Una de las impresiones se resume a continuación en la Figura 1:

\section{1. ¿Qué te gustaría aprender durante la realización de la asignatura?}

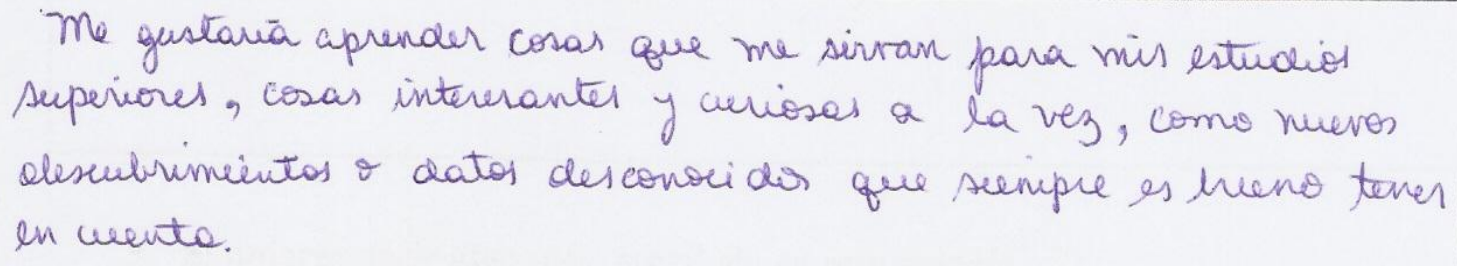

Figura 1: Testimonio de Andrea Muñoz. Colegio San Nicolás de La Serena.

\section{Primeras Aproximaciones al Trabajo Indagatorio.}

Tras la recolección y socialización de impresiones previas, las primeras aproximaciones con los grupos a la metodología, consideraron temas amplios en ecología y se desarrollaron por medio de investigaciones guiadas dentro de los límites del patio de la escuela. Tales investigaciones y las posteriormente ejecutadas ya de forma más autónoma, siguen la ruta cíclica pregunta-acción-reflexión.
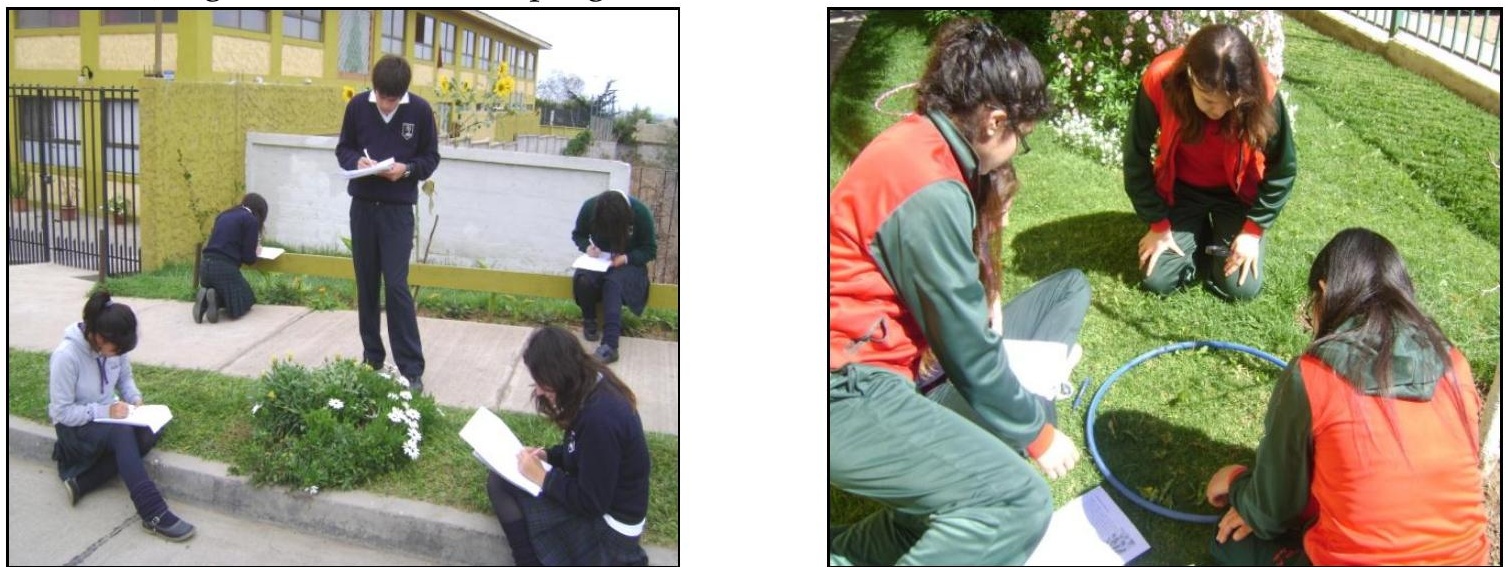

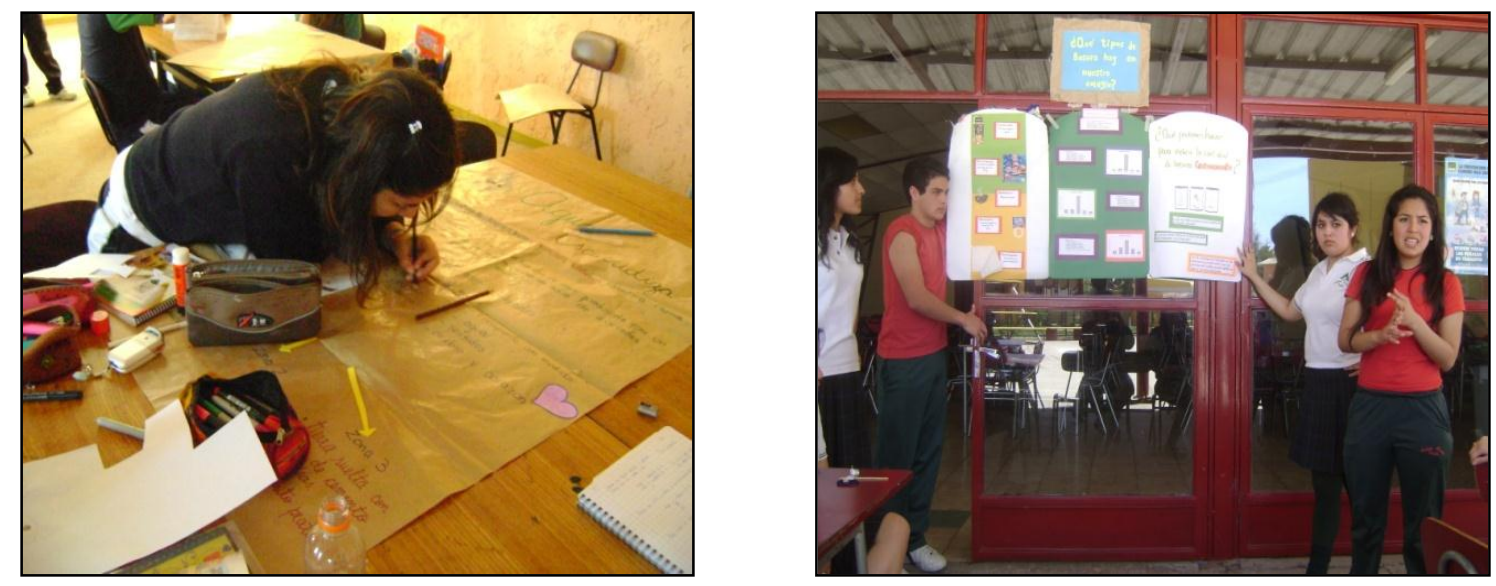

Fotos 5 a 8: Equipos de estudiantes desarrollando sus procesos de investigación guiada.

Temas tales como la erosión del suelo, los roles de la cobertura vegetal, la contaminación del entorno, entre otros, fueron introduciendo a los estudiantes no sólo en las problemáticas medio ambientales, sino que también en las dinámicas de observación, recolección y análisis de datos (etapa de acción) y comunicación-discusión de resultados y conclusiones (etapa de reflexión), propias de la metodología empleada (ver fotografías 5 a 8). En esta fase, la pregunta indagatoria, era previamente definida, discutiendo su estructura y validez a lo largo del desarrollo de las secuencias de investigación.

Una vez finalizados varios ejercicios introductorios, se pasó a la etapa en donde los propios equipos debieron plantear indagaciones en base a los contenidos fijados para cada unidad. En lo que concierne a la práctica pedagógica, se colocó el énfasis en dos aspectos: poder congeniar contenidos del área de la ecología con el entorno local inmediato y facilitar el desarrollo de una secuencia lógica de estudios de primera mano.

Para lograr esto, se desarrollaron visitas a los entornos naturales de los establecimientos, a saber, el Parque Urbano del Campus Andrés Bello de la Universidad de La Serena y la Quebrada El Ingenio, en Ovalle. El objetivo de estas visitas era que los distintos grupos de investigación, pudieran contrastar los contenidos teóricos construidos en clases con la realidad paisajística que ofrecían cada uno de los sitios y así diseñar y planificar sus respectivos procesos de recogida de datos.

\section{Investigaciones en el Entorno Local.}


Resulta común escuchar desde diversos sectores, incluidos los estudiantes, la necesidad de romper con las pautas tradicionales en las que se imparte la ciencia a nivel escolar, más cuando se trata de la Biología, ciencia más ligada al entorno natural que a los muros de la escuela. En este contexto, Guisasola, et al. (2005) señalan que la enseñanza escolar de la ciencia reconoce que los programas de estudio necesitan del acceso al mundo más allá de las aulas.

Es por esto que las excursiones realizadas con vista a resolver problemas y a recoger información y elementos de apreciación, constituyen una parte importante en la enseñanza elemental de la ciencias (UNESCO, 1966). Teniendo en cuenta que los procesos de aprendizaje en contextos tales como un bosque o una playa, son diferentes en muchos aspectos de aquellos asociados con la escuela, esta experiencia pedagógica consideró la hipótesis descrita por Castro (2005), el cual nos plantea que:

"si se propicia en los estudiantes la necesidad de indagar un aspecto de su entorno natural (de manera colectiva) y de socializar los aspectos fundamentales de su trabajo; entonces ellos accederán a otros modos de conocer y desarrollarán particulares formas de investigar".

La puesta a prueba de esta hipótesis nos lleva a un necesario cuestionamiento de la planificación del trabajo de terreno en ciencias naturales, ya que si se logra implementar trabajos de campo sistematizados, basados en la promoción de habilidades y actitudes como movilizadores de conocimientos, podríamos aspirar a una alfabetización científica más efectiva en los jóvenes.

Es así que enfrentados los estudiantes a preguntas sobre los principios de indagación naturalista, los cuales fueron previamente discutidos, estos manifestaron ciertos elementos comunes reflejados en este testimonio que sigue:

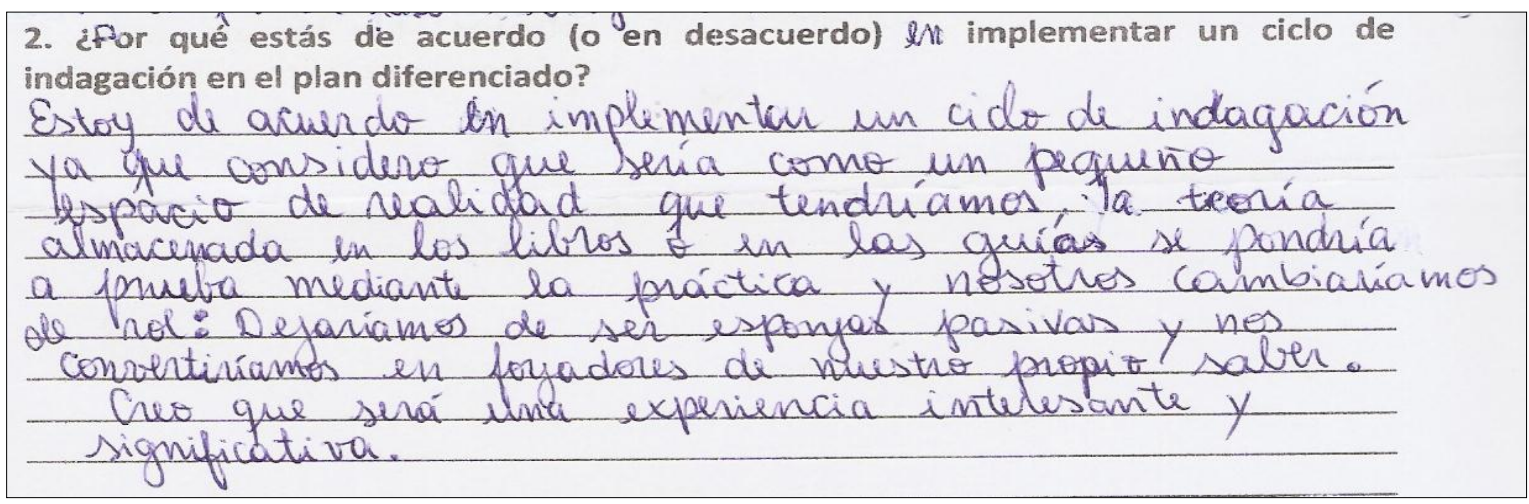

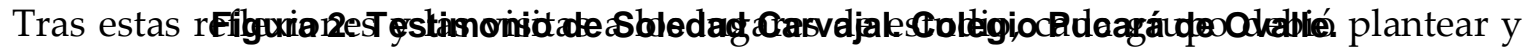
definir una pregunta indagatoria, una acción para dar respuesta a esta y finalmente definir algunos parámetros para realizar la reflexión o análisis de sus respectivos resultados. Sin duda el planteamiento de la pregunta, fue uno de los ítems más complejos, pues por un lado debía relacionarse con los temas propuestos por la unidad de aprendizaje y por otro, debía cumplir con los parámetro que se muestran en la tabla 1, 


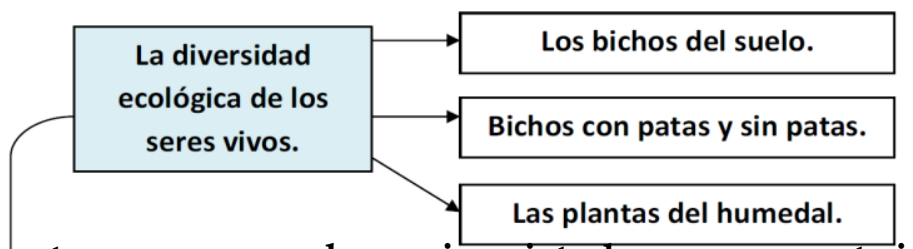

Tabla 1: Parámetros para pasar de una inquietud a una pregunta indagatoria.

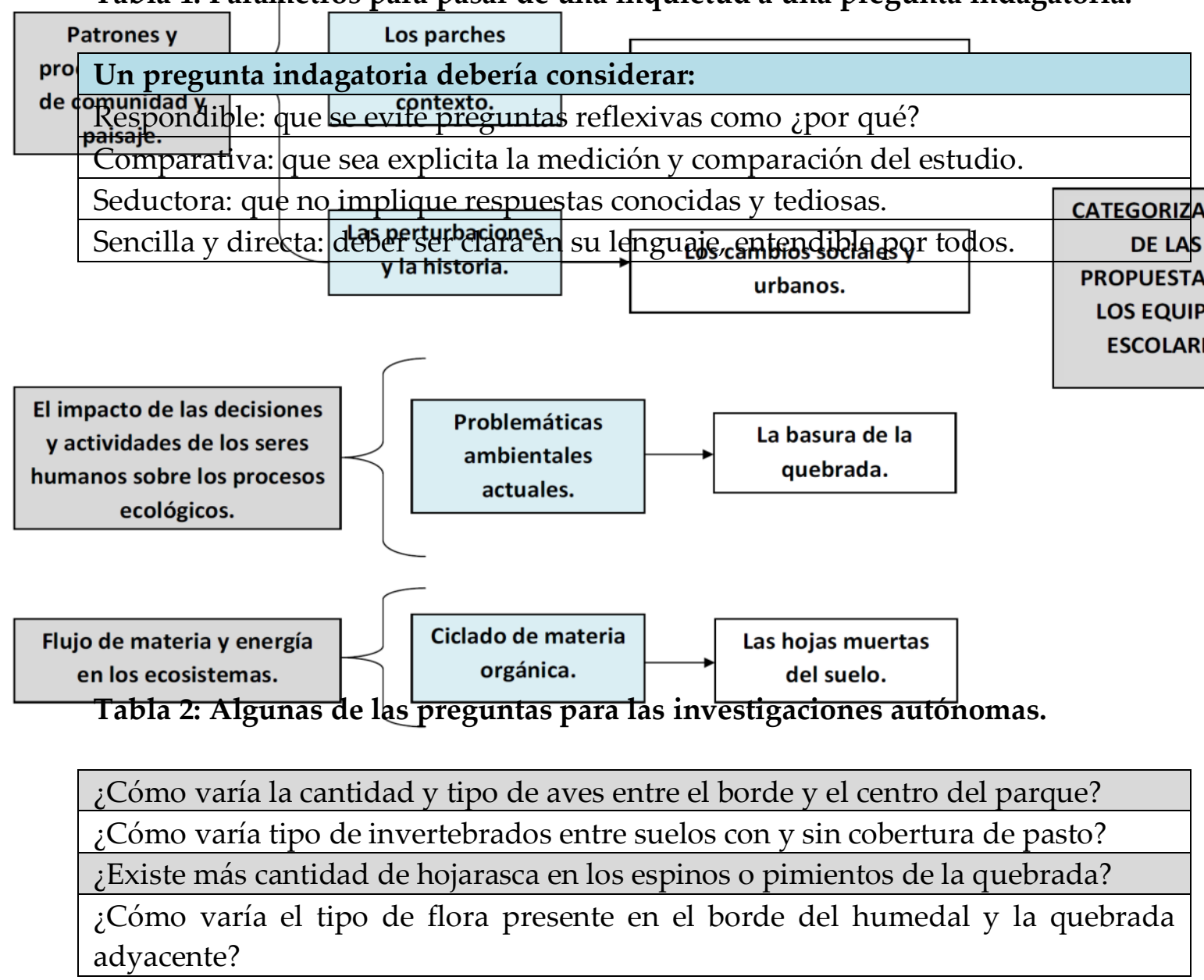

y que la diferencian de una inquietud, siguiendo de forma sintética lo planteado por Arango et al. (2002).

La distinción de que se va a medir y que se va a comparar y su redacción como pregunta de indagación, fue problemática al principio del proceso, pues obligó a los estudiantes a cambiar su habitual forma de enfrentar una investigación escolar, basada la mayoría de las veces, en una mera recopilación de información en base a un tema. Esto fue abordado por medio de una discusión guiada para denotar la relevancia de establecer con claridad, pensando en las posteriores acciones de campo, lo que se quiere cuantificar o cualificar (abundancia, masa, tipo, etc.) y las condiciones de comparación para establecer posteriores conclusiones (borde versus el centro de un parque, suelos con o sin cobertura, pasado y presente, etc.). Los resultados de este proceso se muestran en la tabla 2. 
A modo de síntesis, en el esquema 1 se presentan las líneas de investigación desarrolladas por los estudiantes, categorizadas en base al esquema conceptual propuesto por Rozzi et al. (2000) en torno a los temas aptos para investigaciones ecológicas en el entorno cotidiano (en los recuadros blancos los temas explorados por los equipos escolares).

Luego de la socialización de las propuestas de indagación, los grupos procedieron a levantar los datos en terreno. Varias fueron las clases destinadas al trabajo de campo, en donde se censaron aves, realizaron colectas botánicas, entrevistas, recolección de muestras bióticas y abióticas, consultas a científicos/as, etc. (ver fotografías 9 a 12).

Por lo demás, la adecuación de los métodos a las contingencias de los distintos territorios, se manifestaron en ambos ciclos de enseñanza. Es así como la lluvia, típica por las mañanas de la ciudad de La Serena, altero más de una vez la metodología para la colecta de artrópodos caminantes. También un incendio en la zona de estudio de Ovalle, alteró las zonas de muestreo de plantas del humedal. Sin embargo, esto último dio pie para problematizar las consecuencias del accionar humano en los ecosistemas.

Regularmente se procedió a mostrar estados de avance de las investigaciones. Adicionalmente, el grupo de $4^{\underline{0}}$ año medio, en base a los aprendizajes esperados definidos curricularmente para el nivel, fue guiado además en la redacción de un comunicado escrito con las respectivas etapas de investigación, siguiendo un formato estándar. 

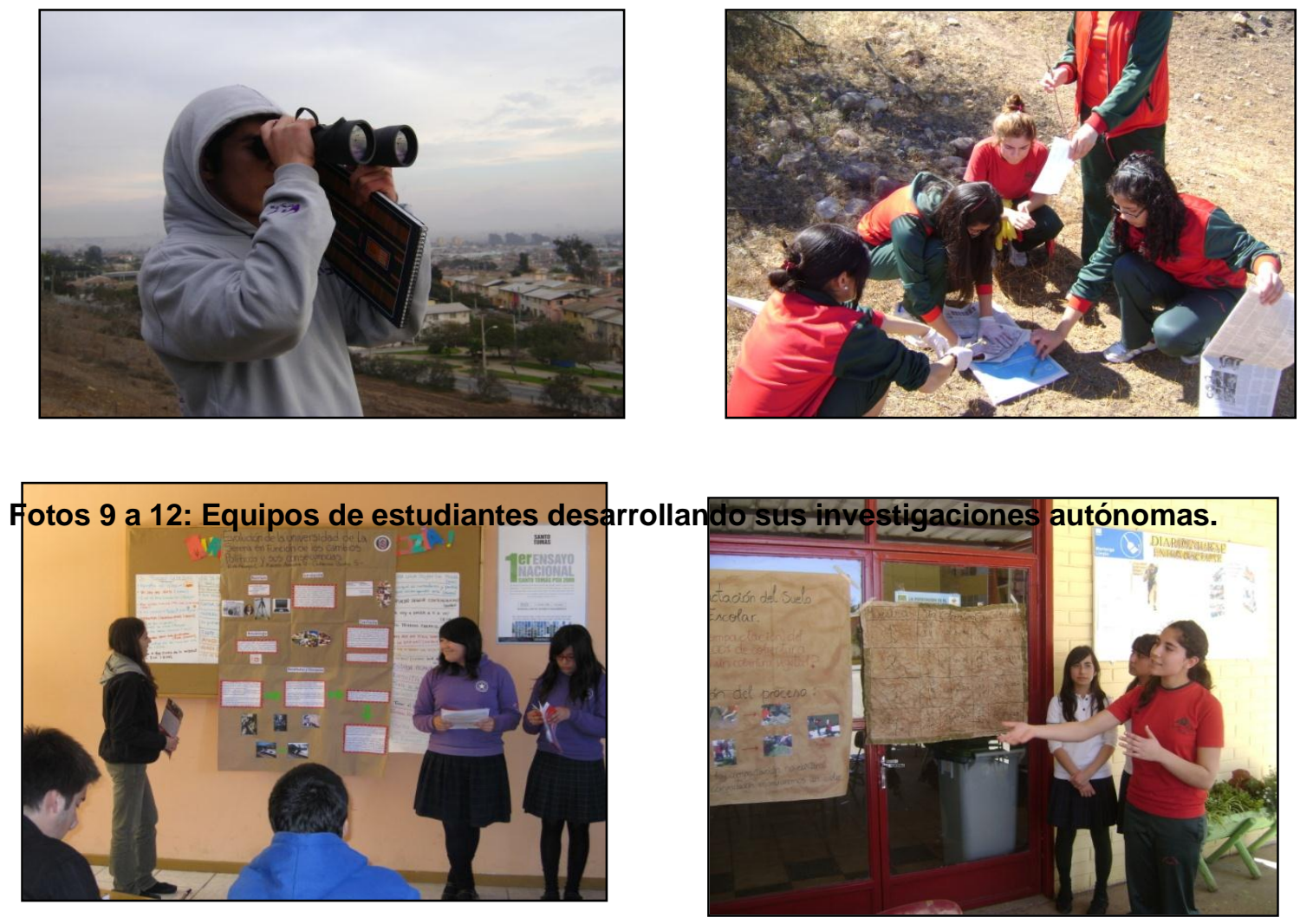

\section{Comunicación del Proceso Científico.}

Pensando en la validación que se hace tradicionalmente del conocimiento científico, se ejecutaron una serie de instancias de comunicación de resultados. Para esto se utilizaron materiales simples, prescindiendo de instrumentos tecnológicos, como computadoras y proyectores. La filosofía de la EEPE plantea el desafío de no depender de aparatos sofisticados para desarrollar experiencias.

Es así como cada grupo debió emplear el máximo de creatividad y exigir una pertinente cohesión de equipo, para poder plasmar de forma resumida y atractiva cada aspecto de su investigación. Materiales como cartulinas, tijeras, cartón y elementos de desecho, fueron los protagonistas en los paneles que debieron confeccionar los estudiantes. Las evidencias fueron presentados en sesiones denominadas "Congresos Científicos", previa definición de la pauta de evaluación de las presentaciones y de los paneles, tomando como referencia los indicadores tipo definidos por el Programa Explora (2010).

Para el caso de los estudiantes de tercer año medio, estas presentaciones se realizaron entre el grupo de pares. Ampliando esto, los jóvenes de cuarto año, llevaron sus resultados al curso "Metodología de la Enseñanza de la Biología", correspondiente al tercer año de la carrera de Pedagogía en Biología de la Universidad de La Serena. Esta 
acción vino a cerrar el proceso de redacción del artículo de síntesis. Estas instancias sirvieron para generar una rica retroalimentación entre los equipos investigativos y otros evaluadores, con lo cual, en base a pautas de evaluación y discusión libre, se buscaba ejercitar el juicio crítico y la valoración personal y grupal (ver fotografías 9 a 12).

\section{Actividades Complementarias.}

A lo largo de los dos procesos, se buscaron instancias que mostraran distintas facetas de la actividad científica, de manera que los estudiantes notaran, por ejemplo, que la misma no es un trabajo que sólo se realiza en los grandes centros de investigación del mundo, sino que también en la región, existen mujeres y hombres de ciencia. Es así como con el primer grupo, se organizaron instancias en donde científicos locales pudieron transmitir sus conocimientos y experiencias.

También se participó en la Semana Nacional de la Ciencia y Tecnología 2009, que organiza el Programa Explora, dependiente de la Comisión Nacional de Investigación Científica y Tecnológica, CONICYT. En los Laboratorios de Biología de la Universidad de La Serena, pudieron apreciar de forma lúdica distintas áreas de investigación y utilizar instrumental técnico.

Por otro lado, el trabajo con el tercer año coincidió con la designación del 2010 por la Organización de Naciones Unidas, como el Año Internacional de la Biodiversidad Biológica. Variadas acciones se realizaron mundialmente, entre ellas el "Festival Mundial de Aves", evento coordinado por BirdLife International, donde se intenta concentrar esfuerzos a favor de la conservación de las aves y sus ambientes.

Fue así que como equipo organizamos una visita a los humedales costeros de la ciudad de Tongoy (ubicada al norte de Ovalle), en donde se desarrollan distintas iniciativas de conservación ligadas a estos ecosistemas.

Esta visita permitió compartir con un investigador de aves local, el cual apoyo el trabajo práctico, además de la aplicación de una pequeña indagación que por medio de la pregunta ¿cómo varía la riqueza
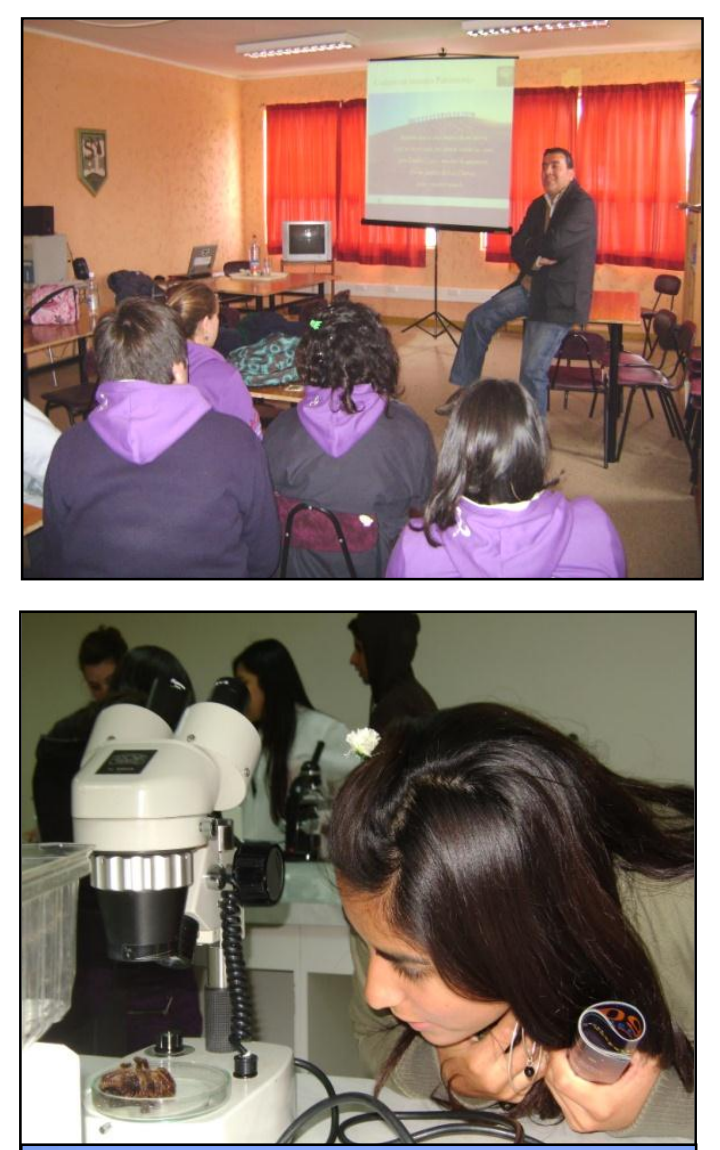

a

a
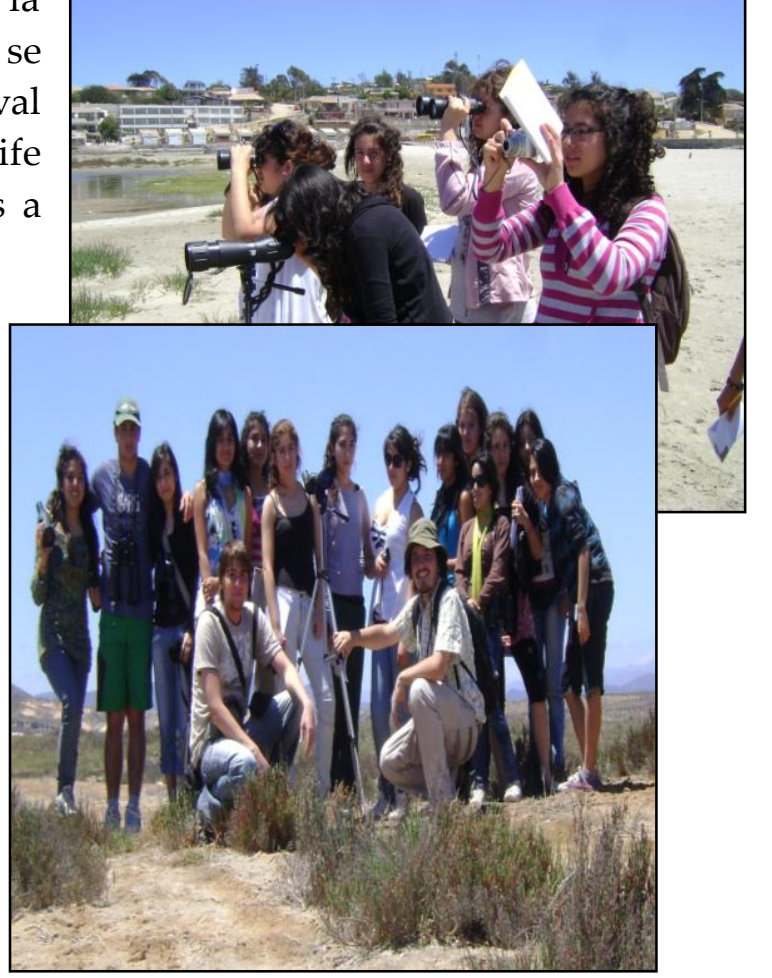

Fotos 13 a 16: Desarrollo de actividades de 
de especies de aves entre los humedales Estero Tongoy y Salinas Chica de la Bahía de Tongoy?, pretendía que los estudiantes se transformaran en ornitólogos y ornitólogas por algunas horas.

Como actividad de cierre, en el humedal de Salinas Chica, los estudiantes redactaron un testimonio denominado "Carta desde el Humedal" (Ministerio del Medio Ambiente, 2009), en donde plasmaron sus impresiones (ver fotografías 13 a 16).

\section{Evaluación de los Procesos.}

Hay que recordar que los jóvenes participantes de este proyecto, por inclinaciones personales eligieron en segundo año medio el electivo de ciencias biológicas. Considerando esto, la definición y cumplimiento de tareas individuales dentro de cada equipo y la propia autorregulación frente al avance de la investigación, fueron desafíos a las tradicionales pautas de comportamiento en la clase de ciencias, que en distintos grados de éxito fueron sorteados. Actitudes como la responsabilidad y el trabajo en equipo y habilidades como la planificación y comunicación de ideas, fueron sin duda las más exigidas durante ambos procesos. Esto no hizo más que hacer reflexionar a cada grupo frente a como la actividad científica, como cualquier otra, no escapa a los problemas que surgen dentro de cualquier grupo humano.

Se constata un cambio en las pautas con las cuales los estudiantes abordan el trabajo científico escolar, apropiándose de una estructura que les posibilita obtener, ordenar (por categorías), analizar (en base a evidencia) y comunicar información pertinente. Esto no tan sólo en ciencias, ya que en base a testimonios y evidencias presentadas por docentes del área de Lenguaje, los estudiantes son capaces de incorporar las herramientas adquiridas en el curso a otras dimensiones de su trabajo escolar. Algunos indicadores de este aspecto, se reflejan en el siguiente testimonio de una alumna de tercer año medio.

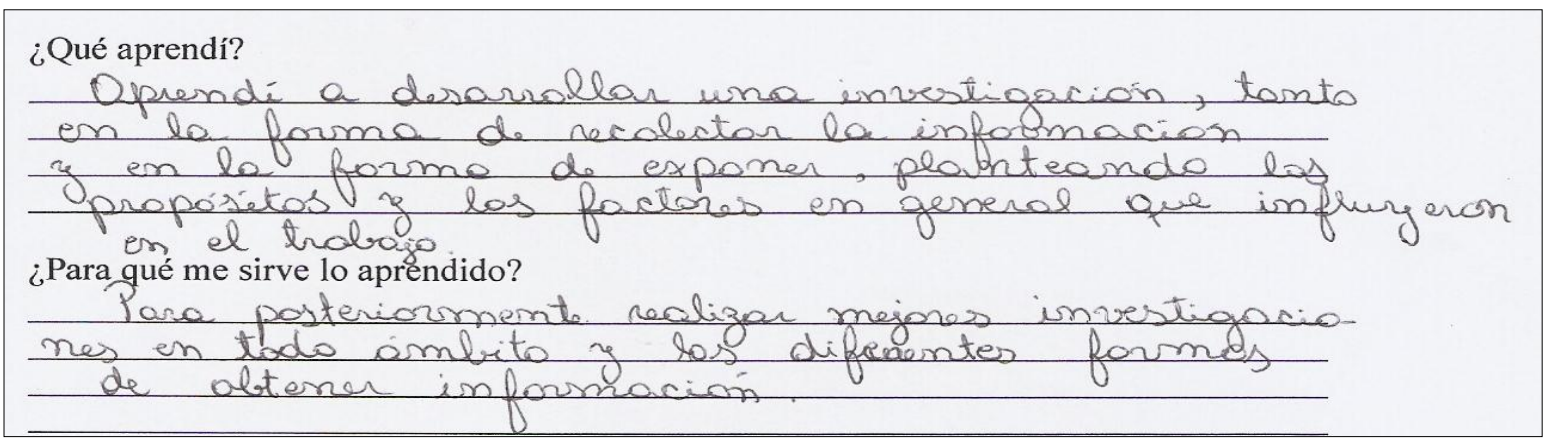

Figura 3: Testimonio de Vaittiare Pizarro. Colegio Pucará de Ovalle. 
Además, y dando cuenta de la hipótesis inicial considerada en este trabajo, el grupo valora y proyecta la utilización futura del conjunto de habilidades y actitudes adquiridas y desarrolladas durante el curso, en pos de sus propias aspiraciones y metas académicas planteadas al inicio del trabajo.

Obtienen datos relevantes sobre cada uno de los temas estudiados, modificando su propio conocimiento sobre el tema, mostrando además una valoración de las evidencias por sobre los juicios apreciativos, gracias a que logran estructurar de forma inédita dentro de sus trayectorias escolares, escritos y presentaciones con un formato acordé en líneas generales a la construcción y comunicación del conocimiento científico.

Finalmente, los estudiantes valoran el entorno local como aula abierta de aprendizaje, mejorando su disposición hacia el estudio y el conocimiento. Disfrutan del contacto al aire libre y hacen explicita esta situación con sus pares y hacia los docentes.

\section{Agradecimientos:}

Agradecer en primer lugar el entusiasmo y sinceridad de mis alumnos y alumnas, cualidades fundamentales para el éxito de este proyecto. A los profesores Carlos Zuleta R, Daniel Hiriart L, Víctor Bravo N. y Geraldo Brown G. por compartir su tiempo y conocimientos con los estudiantes, tanto en terreno como en el aula y a Claudia Hernández P. por entregarme las primeras luces sobre esta metodología.

Finalmente agradezco a la profesora Fanny Angulo D. de la Universidad de Antioquia, Colombia, cuyo apoyo a la distancia y revisión de este manuscrito, ha sido fundamental.

\section{Referencias Bibliográficas}

Arango, N. et al. (2002). Guía metodológica para la enseñanza de la ecología en el patio de la escuela. National Audubon Society. Nueva York, USA,

Arango, N. et al. (2009). Principios y prácticas de la enseñanza de la ecología en el patio de la escuela. Instituto de Ecología y Biodiversidad - Fundación Senda Darwin. Santiago, Chile. 
Castro, J.A. (2005). La investigación del entorno natural: Una estrategia para la enseñanza-aprendizaje de las ciencias naturales. Universidad Pedagógica Nacional. Bogotá, Colombia.

Chile, Ministerio de Educación. (2002). Biología. Programa de estudio cuarto año medio formación diferenciada. Unidad de currículum y evaluación. Ministerio de Educación. Santiago: Editor.

Chile, Ministerio de Educación. (2002). Biología. Programa de estudio tercer año medio formación diferenciada. Unidad de currículum y evaluación. Ministerio de Educación. Santiago: Editor.

Chile, Ministerio de Educación. (2005). Marco curricular objetivos fundamentales y contenidos mínimos obligatorios de la educación media. Unidad de currículum y evaluación. Ministerio de Educación. Santiago: Editor.

Chile, Ministerio del Medio Ambiente. (2009). Guía educativa los humedales: Espacios para conservar y disfrutar. Departamento de educación ambiental y participación. Ministerio del Medio Ambiente. Santiago: Editor.

Guisasola, J. et al. (2005). Diseño de estrategias centradas en el aprendizaje para las visitas escolares a los museos de ciencias. [Versión electrónica]. Revista Eureka sobre Enseñanza y Divulgación de las Ciencias. Vol. 2, № 1, 19-32.

Piñones, C. (2010). Resituando la actividad científica en la escuela: Investigaciones escolares en ecología dentro del entorno urbano local. Ponencia presentada en el Primer Encuentro Nacional de Divulgadores Científicos, Valdivia, Chile.

Programa Explora. (2010). Guía de apoyo a la investigación científica escolar. Comisión Nacional de Investigación Científica y Tecnológica. Santiago, Chile: Editor.

Rozzi, R. et al. (2000). La enseñanza de la ecología en el entorno cotidiano. Programa MECE-MEDIA, Ministerio de Educación. Santiago, Chile.

Organización de las Naciones Unidas. (1966). Manual de la Unesco para la enseñanza de las ciencias. ( $3^{\circ}$ ed.). Organización de las Naciones Unidas. Editorial Sudamericana, Buenos Aires, Argentina. 